
زنوتيبهاى برنج تحت تنش كمبود نيتروزن

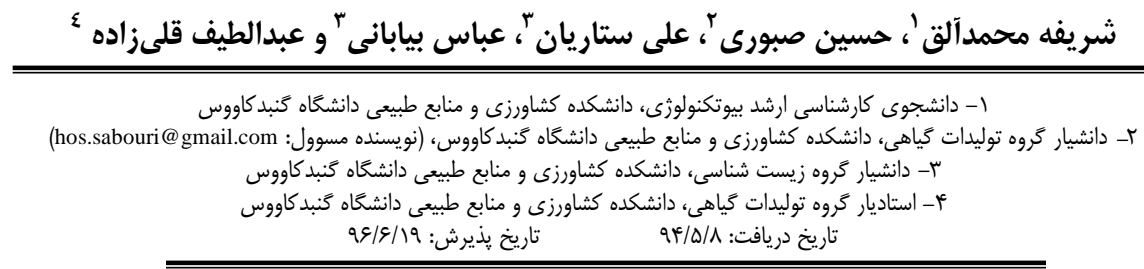

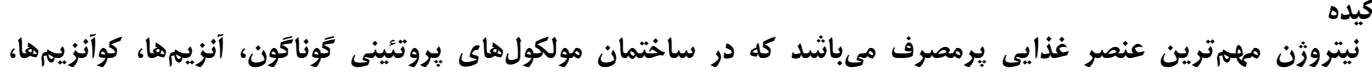

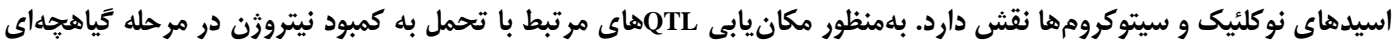

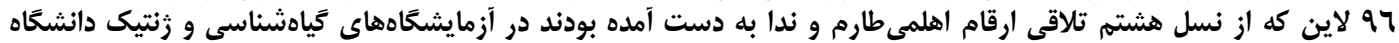

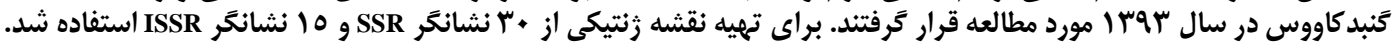

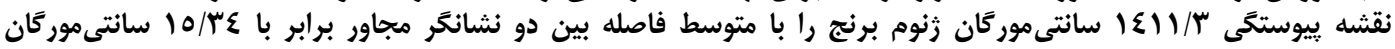

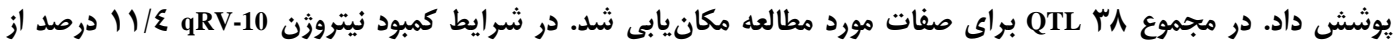

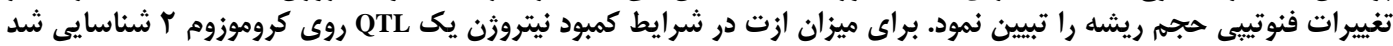

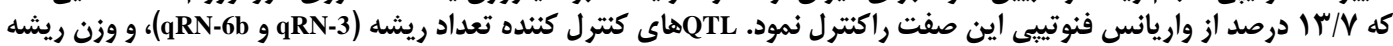

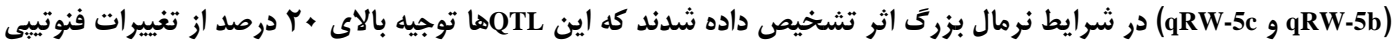

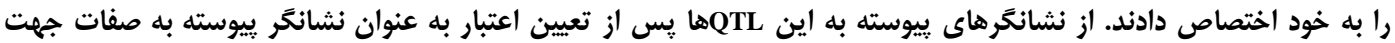

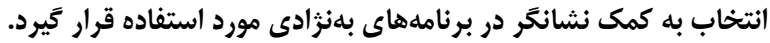

وازههاى كليدى: برنج، مرحله تَياهُهاى، كمبود نيتروزن، مكان يابى صفات كمى

بين واريتههاى TN1 (هندى) و CJ06 (ثاينى) توانستند در

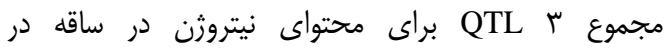

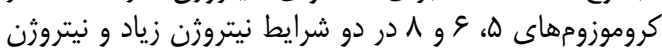

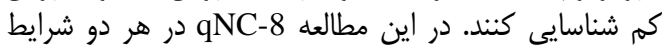
در فاصله نشانكرى

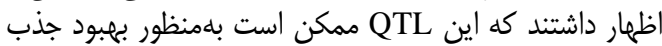

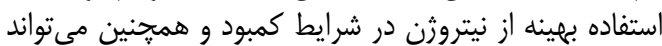

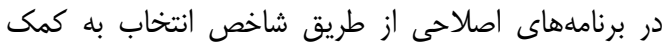

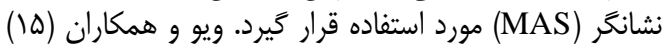

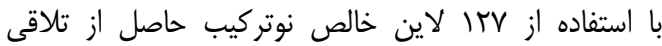

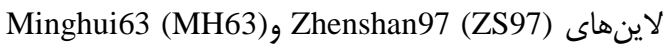
به همراه والدين و نسل F1 توانستند در شرايط كمبود نيتروزن

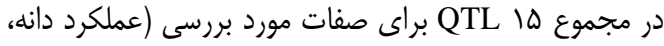

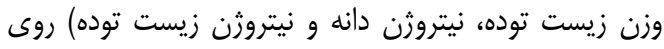

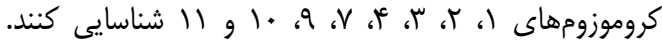

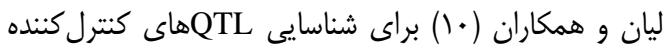

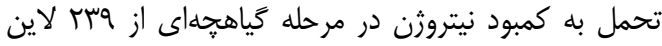

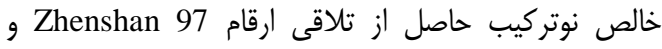
Minghui 63

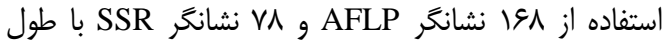

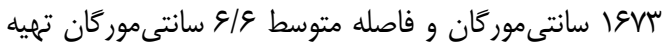

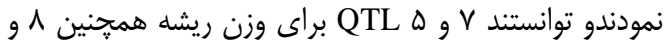

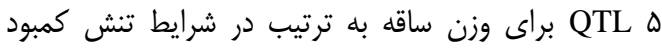

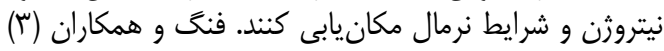

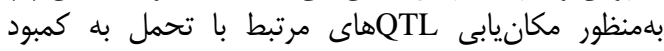

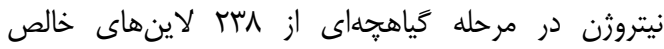
نوتركيب حاصل از تلاقى Rieqingzao B

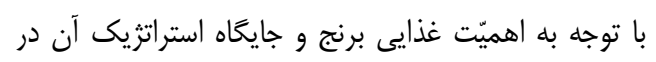

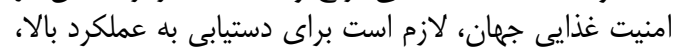

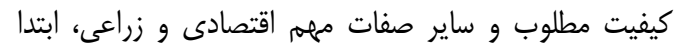

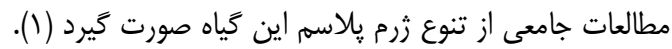

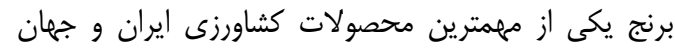

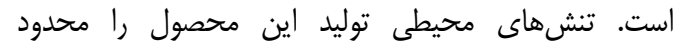

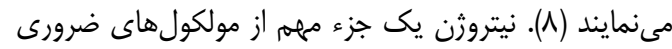

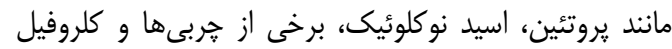

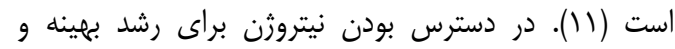

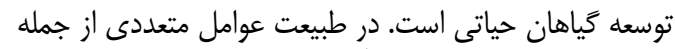

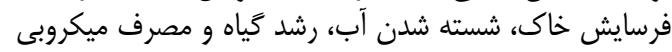

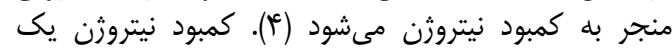

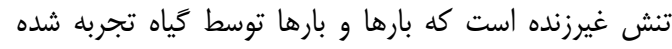

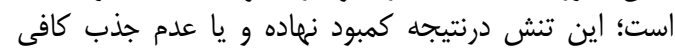

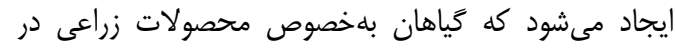

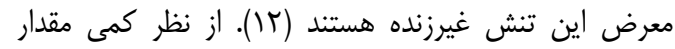

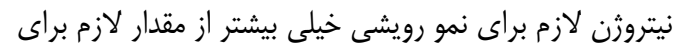

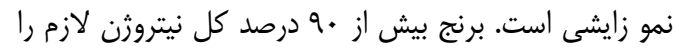

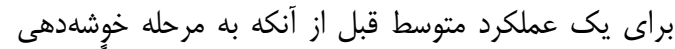

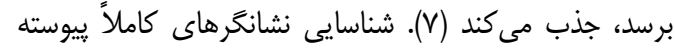

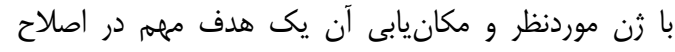

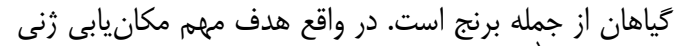

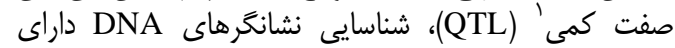

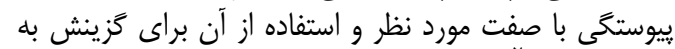

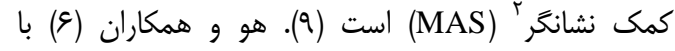

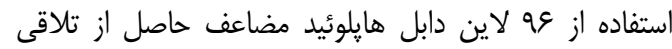


شد. صفات طول ساقه و ريشه، طول و عرض برك يرحته با

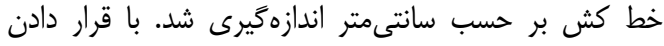

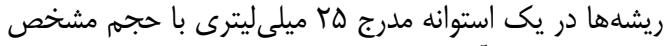

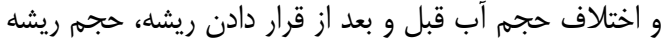

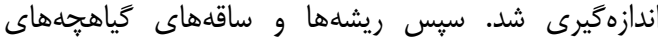

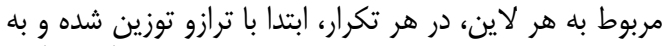

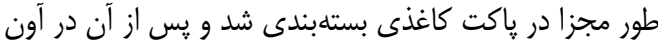

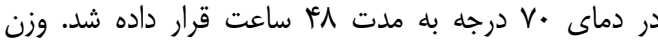

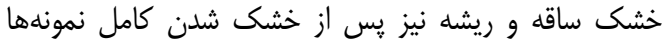

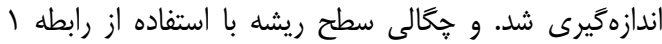

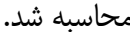

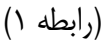

(T)

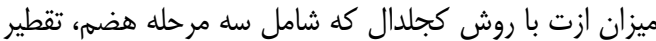

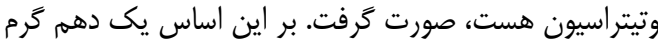

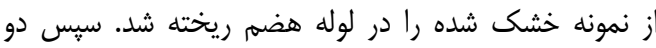

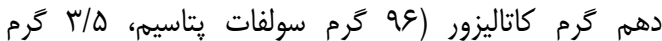

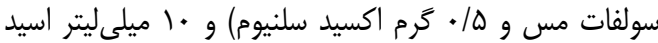

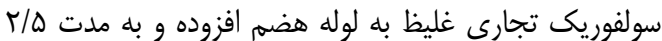

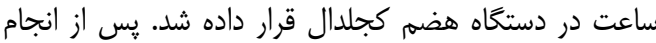

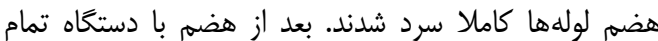

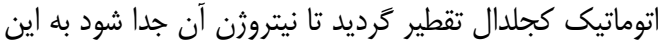

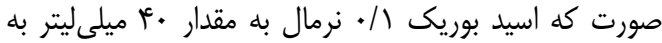

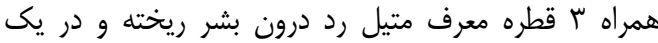

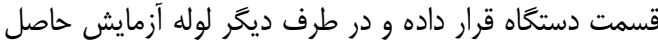

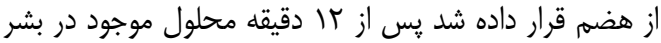

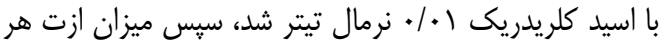

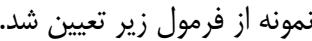

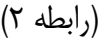

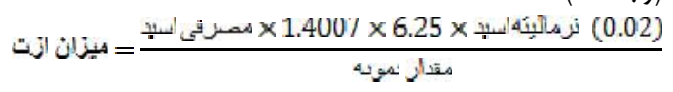

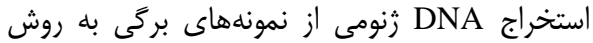

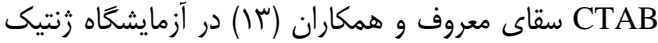

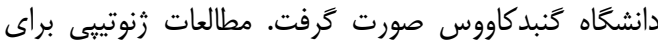

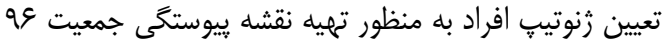

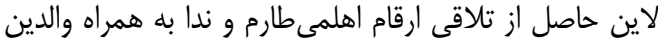

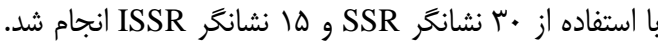

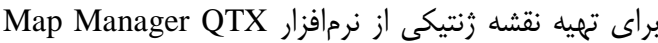

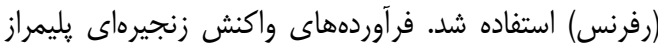

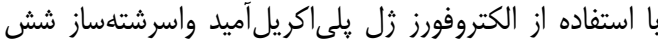

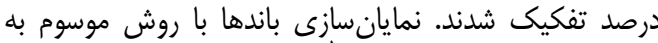

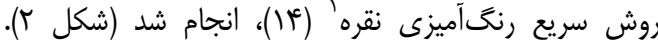

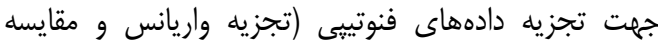

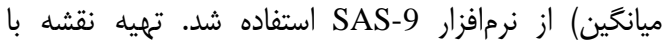

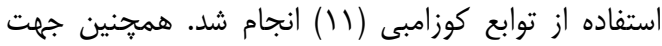

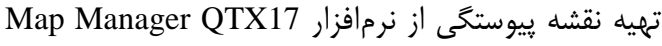

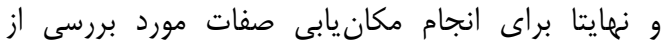

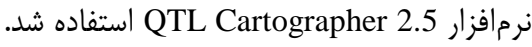

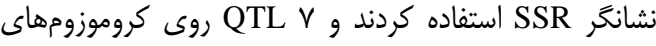

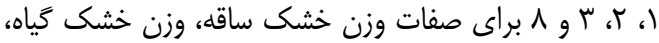

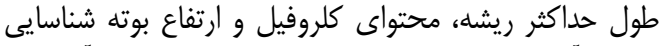

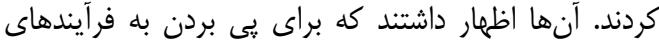

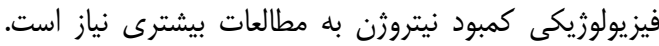

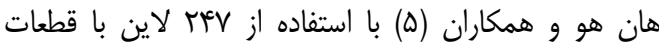

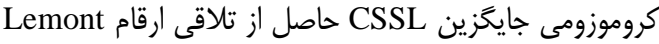

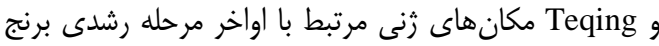

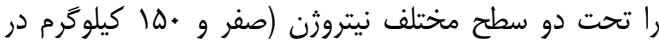

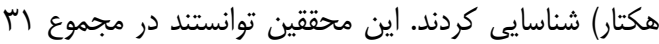

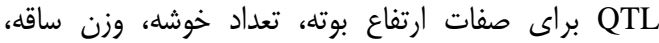
محتواى كلروفيل و عملكرد دانه شناسايى كنند. يو و همكارات

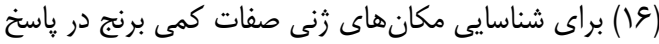

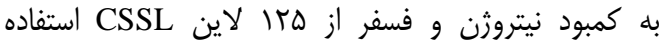

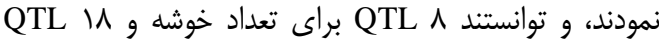

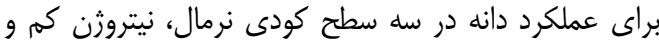

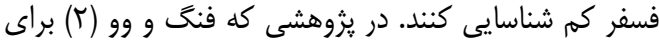

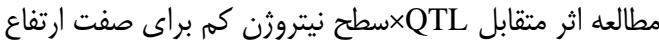

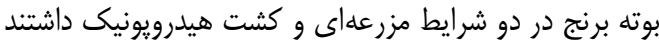

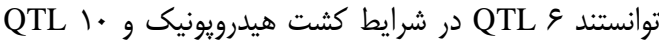

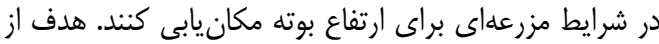

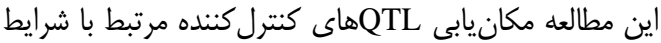

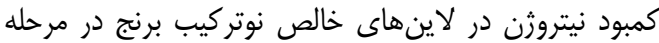
كياهجهاى بود نيترون

\section{مواد و روشها}

اين آزمايش به صورت كات كشت هيدرويونيك در سال سوسا

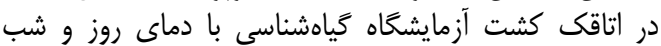

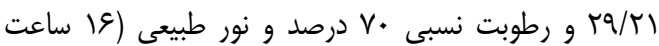

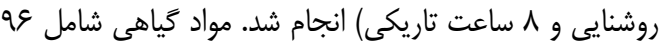

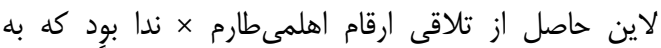

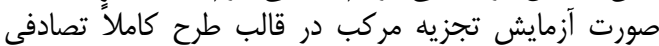

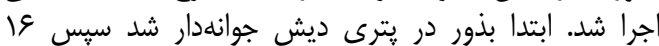

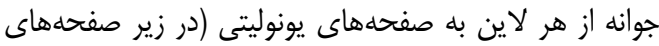

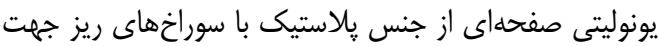

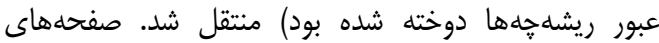

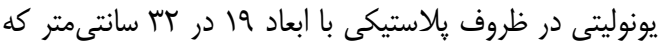

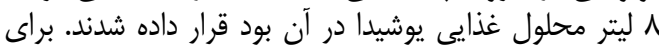

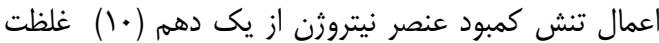

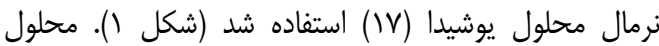

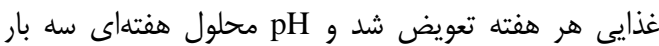

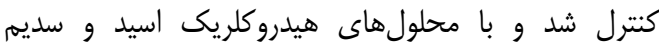

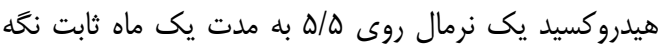

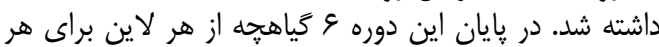

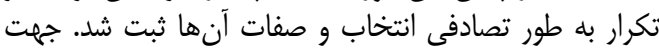

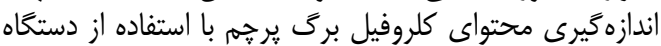

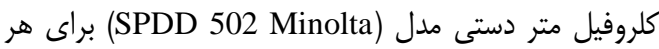

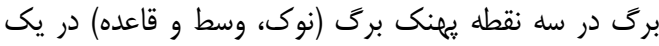
سوى رگبرگ اصلى قرائت شد. تعداد ريشه نيز شمارش و و ثبت

1- Rapid Silver Staining 


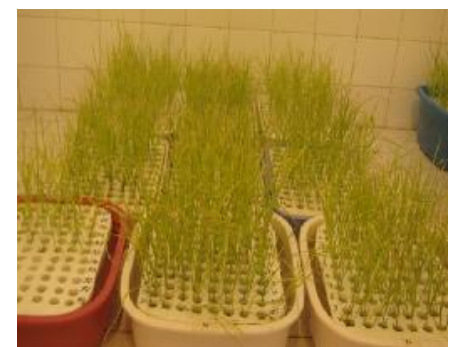

(ب)

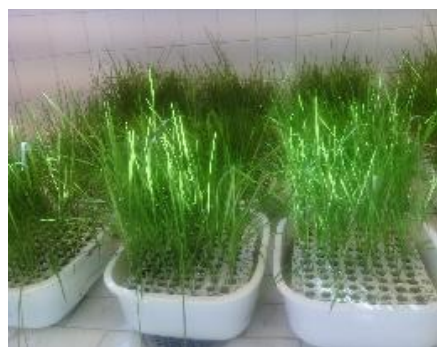

(الف)

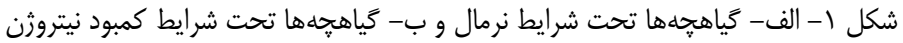

Figure 1. A: Seedlings under normal conditions and B: Seedlings under nitrogen deficiency conditions

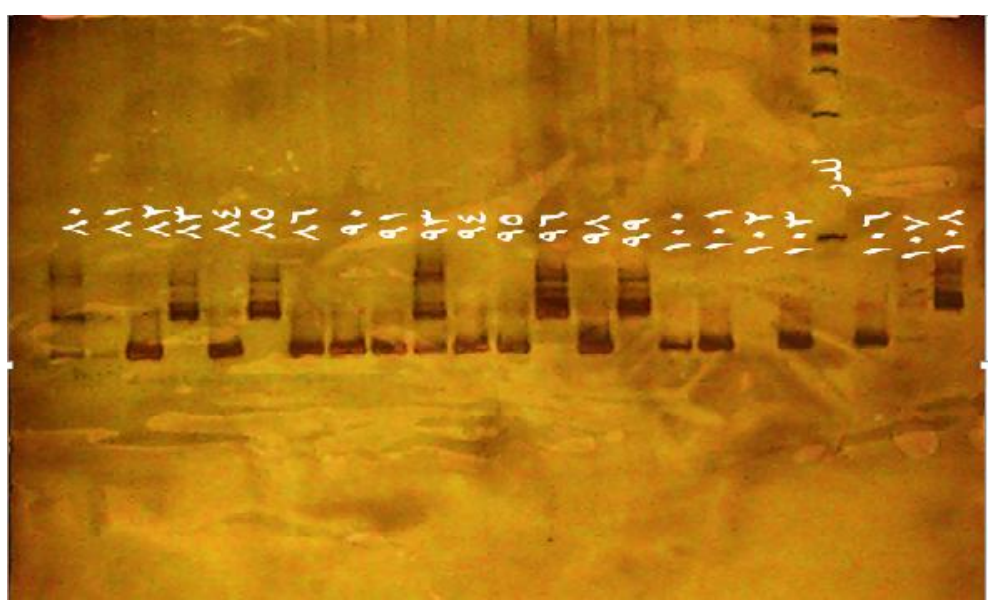

شكل r- الخوى نواربندى با استفاده زل يلى اكريلاميد

Figure 2. Banding patterns using polyacrylamide gel

صفات جَّالى سطح ريشه كه در سطح احتمال ينج درصد

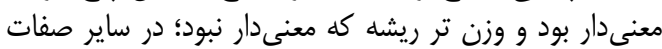

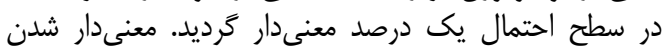

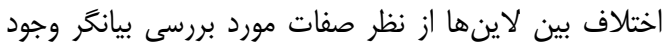

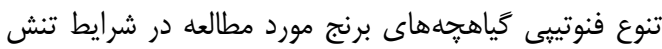

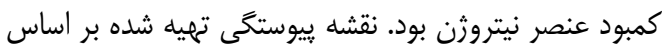

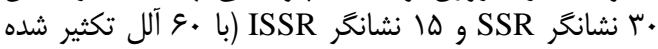

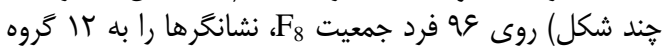

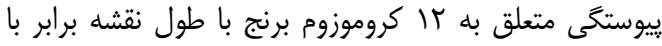

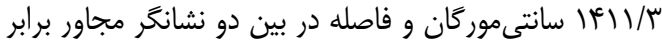

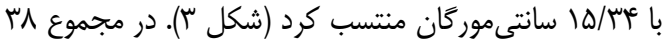

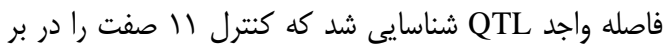

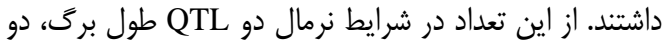

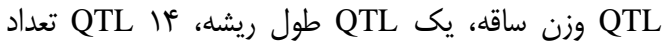

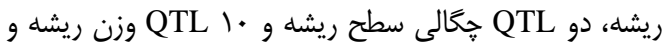

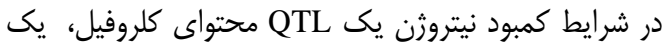

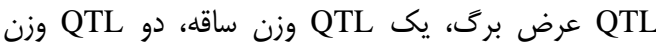
خشك ساقه، يك QTL حجم ريشه و يك QTL
نتايج و بحث

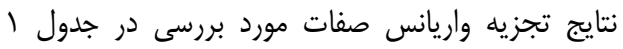

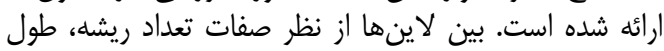

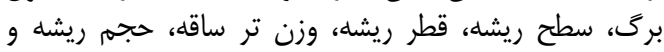

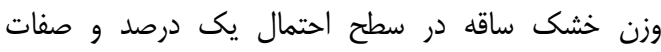

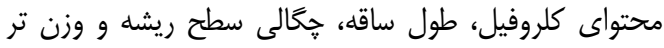

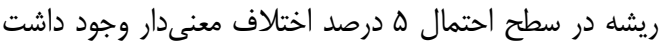

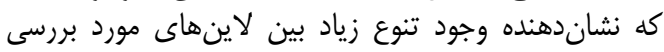

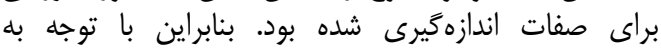

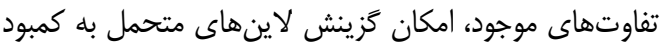

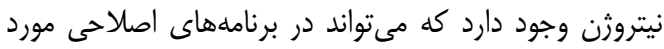

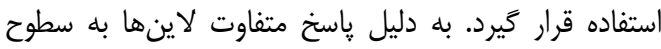

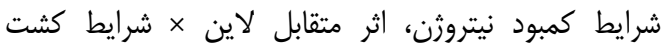

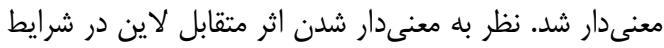

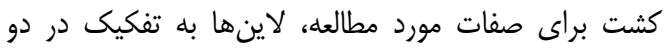

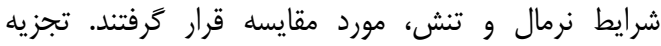

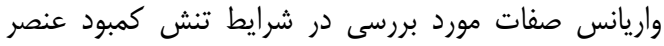

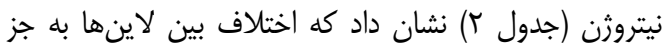




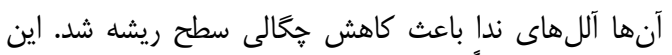

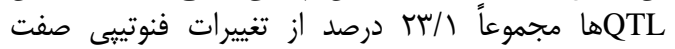

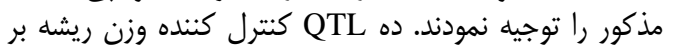

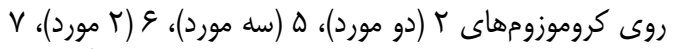

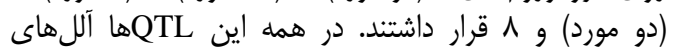

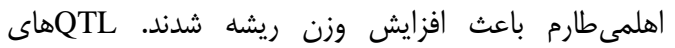

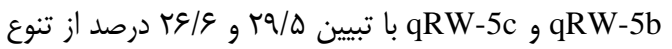

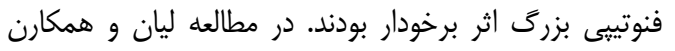

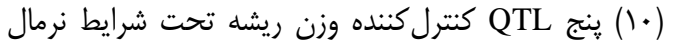

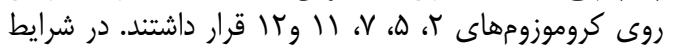

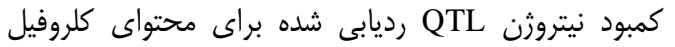

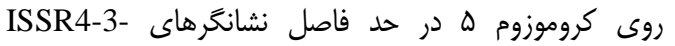

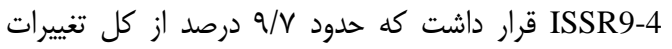

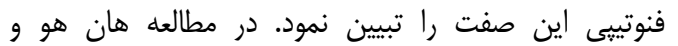

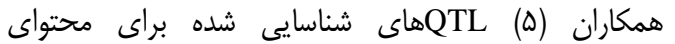

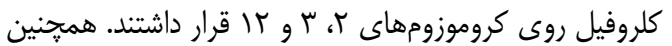

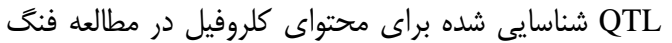

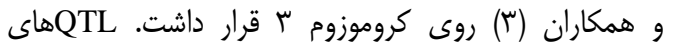

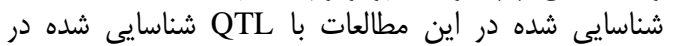

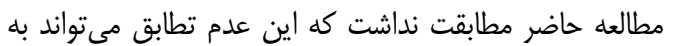

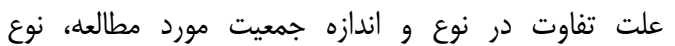

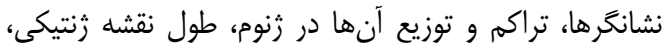

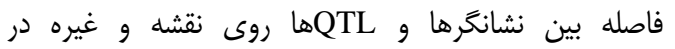

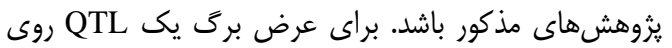

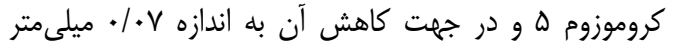

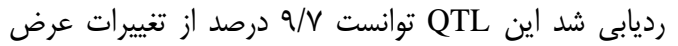

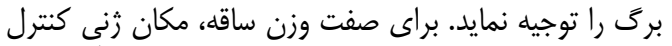

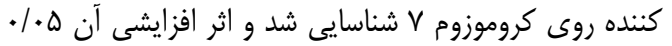

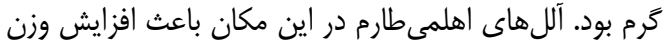

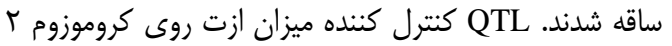

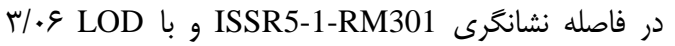

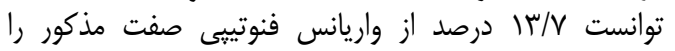
توجيه نمايد.
را كنترل نمودند (جدول rا r). در شرايط نرمال براى طول بروى

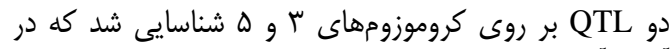

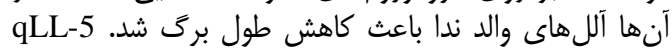

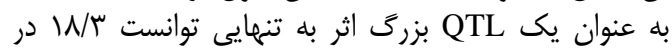

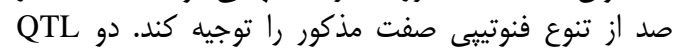

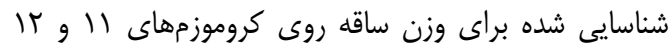

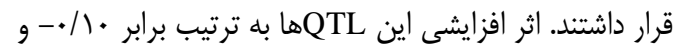

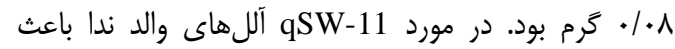

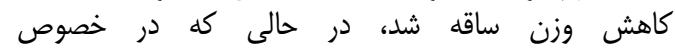
دSW-12 آللهاى اهلمى طارم باعث افزايش وزن ساقل داقه شد.

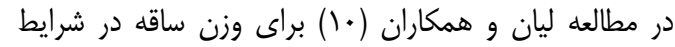

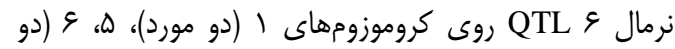

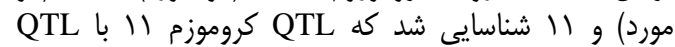

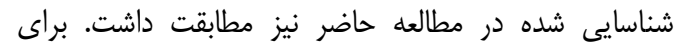

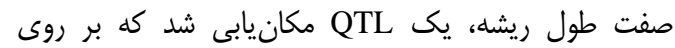

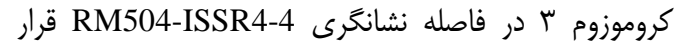
داشت. در اين QTL آللهاى والد اهلمى طارم باعث افزايش

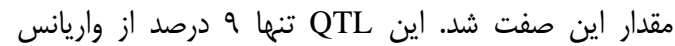

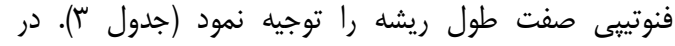

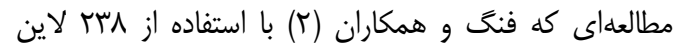

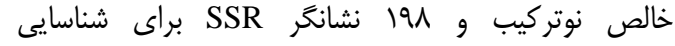
هاى مرتبط با كمبود نيتروثن در مرحله كياهجاهاى

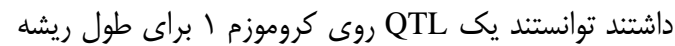

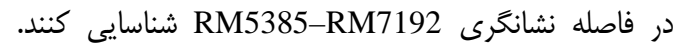

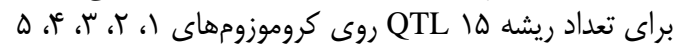

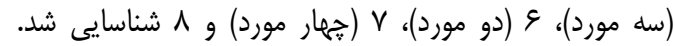

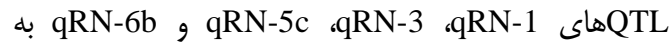

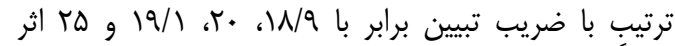

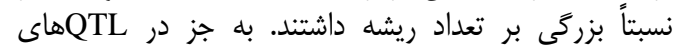

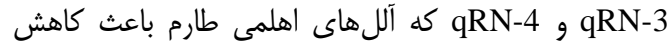

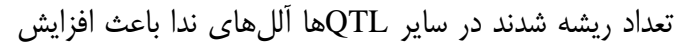

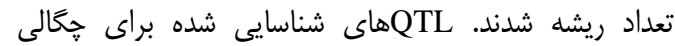

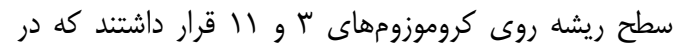




\begin{tabular}{|c|c|c|c|c|c|c|c|c|c|c|c|c|c|c|c|c|}
\hline \multicolumn{15}{|c|}{ ميانكين مربعات } & \multirow[b]{2}{*}{$\frac{\hat{s}}{\overline{\bar{a}}}$} & \multirow[b]{2}{*}{$\begin{array}{l}3 \\
2 \\
\vdots 3 \\
\vdots 3\end{array}$} \\
\hline 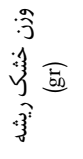 & 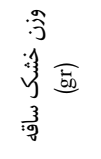 & 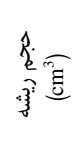 & 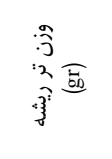 & 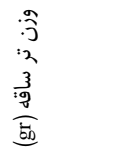 & $\begin{array}{l}\frac{10}{y} \\
\frac{3}{3} \\
\text { ते }\end{array}$ & 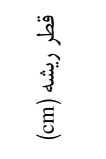 & $\begin{array}{l}3 \\
\frac{3}{2} \\
\frac{3}{3} \\
3\end{array}$ & $\frac{3}{2}$ & $\begin{array}{l}y^{y} \\
3^{2} \\
y^{2} \\
\frac{y^{2}}{E}\end{array}$ & 秀 & $\frac{3}{\frac{3}{n}}$ & 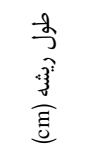 & $\begin{array}{l}\frac{2}{3} \\
\frac{3}{9} \\
\text { 吾 }\end{array}$ & $\begin{array}{l}\frac{3}{2} \\
\frac{2}{y} \\
\frac{y}{2} \\
\frac{3}{3} \\
3\end{array}$ & & \\
\hline$r / \Delta q^{q u t h}$ & $I V / T^{\mathrm{ns}}$ & $\mid V / \Gamma^{* * *}$ & $\cdot / \cdot 9^{\mathrm{nS}}$ & 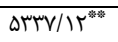 & $1 / \cdot \mu^{* 3}$ & $\cdot / \cdot 19^{* * *}$ & $r q / \mu . * *$ & rq."**: &.$/ 19^{* 1 / 2}$ & $\varepsilon \Delta / \Delta \phi^{* *}$ & $\cdot / \cdot 1^{\mathrm{ns}}$ & $r / 9 q^{* * n}$ & $\varphi \cdot / F \Delta^{* * *}$ & $\cdot(\cdots)^{\mathrm{ns}}$ & $r$ & بلوى \\
\hline$r / \Lambda^{q * * w}$ & $\mid V \Psi / \Gamma^{*}$ & $1 / 9 . *$ & $\mathrm{ragv} / \mathrm{Ve}^{\mathrm{ns}}$ & 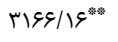 & $\cdot / 4 e^{* 3 *}$ & $\cdot / \omega \Gamma^{* * *}$ & $\mid r / T V^{* * n}$ & $\Delta \Lambda .^{* * *}$ & $1 / \cdot e^{* * * n}$ & $\Delta \mathrm{V} / \mathrm{q}^{* *}$ & $.1 .9^{\text {wat }}$ & 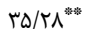 & $\Lambda 1 / \leftarrow \Delta \Delta^{* *}$ & $.1 . \cdot r^{*}$ & 90 & لاين \\
\hline .119 & $91 / 19$ &.$/ \mathrm{V}$ & METI/AF & $1.9 / 99$ &.$/ T \wedge$ &.$/ . \cdot r$ & $1 / \pi F$ & $1 \pi / 98$ & $.1 \cdot 1$ & $F / \pi$ &.$/ . .1$ & ( & T/QT & ...r & 19. & خطا \\
\hline $\mid r / r r$ & YN/MN & $19 / 98$ & $V E / q 1$ & $N / A r$ & $V T / 4 \varepsilon$ & 181.9 & $\mid F / \Delta F$ & سאוא 11/ & $\Delta$ & IV/FT & $N / \cdot r$ & $9 / T V$ & $1 . / \pi 1$ & SV/AT & يرات & ضريب \\
\hline
\end{tabular}

Table 1. The combined analysis of the traits in rice $\mathrm{F}_{8}$ lines under norma

\begin{tabular}{|c|c|c|c|c|c|c|c|c|c|c|c|c|c|c|c|c|}
\hline 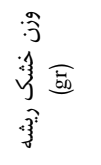 & 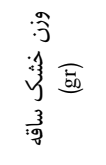 & 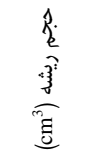 & 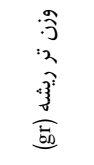 & 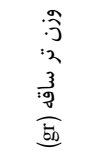 & 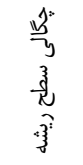 & $\frac{\sqrt[3]{2}}{\frac{3}{3}}$ & $\begin{array}{l}\frac{3}{2} \\
\frac{3}{3} \\
\frac{3}{3}\end{array}$ & $\begin{array}{l}\frac{3}{3} \\
y^{2} \\
y^{2}\end{array}$ & $\begin{array}{l}y^{y} \\
3^{2} \\
y^{2} \\
\frac{8}{0}\end{array}$ & $\begin{array}{l}\frac{g}{j} \\
y^{2} \\
\text { है }\end{array}$ & $\frac{\frac{3}{2}}{\frac{3}{3}}$ & $\begin{array}{l}\frac{-2}{3} \\
\frac{7}{3} \\
\frac{\sqrt[3]{3}}{5}\end{array}$ & 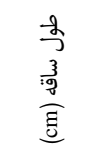 & $\begin{array}{l}3 \\
\frac{3}{y} \\
y \\
\frac{4}{2} \\
3 \\
3\end{array}$ & $\frac{\hat{y}}{\frac{\bar{m}}{\bar{y}}}$ & $\begin{array}{l}\frac{3}{2} \\
\vdots 3 \\
\vdots 3\end{array}$ \\
\hline$\cdot(\cdots)^{\text {ns }}$ & $\cdot / \cdots 0^{* * n}$ & $\cdot / \cdot 1^{\text {twan }}$ & $\cdot / \cdot r^{m}$ & $\cdot / \cdot r^{\text {wits }}$ & $.19 . "$ & $\cdot / \cdots 0^{\text {wth }}$ & $\cdot / r \omega^{* a}$ & $1^{\mathrm{ns}}$ & $\cdot / \ldots r^{\mathrm{ns}}$ & $\cdot / T V^{* * a n}$ & $\cdot / \cdot r \omega^{* *}$ & $\cdot / \cdot r^{\mathrm{ns}}$ & $\cdot / 1 \cdot r^{*}$ & $\cdot\left(\cdot .\left.\right|^{*}\right.$ & $r$ & تكرار \\
\hline$\cdot 1 \cdots 9^{* * *}$ & $\cdot / \cdot \Lambda^{* * *}$ & $\cdot / \cdot 1^{\text {wat }}$ & $\cdot / \cdot \Delta^{*}$ & $F / v \cdot{ }^{* * w}$ & $\cdot / 1^{\mathrm{ns}}$ & $\cdot|\cdots|^{* w a}$ & $\cdot / 1 e^{* \infty}$ & 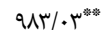 & $\cdot / \Lambda r^{* \infty}$ & $r N /\left.r\right|^{* * *}$ & $|1 / \Delta|^{* *}$ & $1 / \kappa r^{* *}$ & $9 \cdot / 48^{* \text { wath }}$ & $\cdot|0| \cdot$ & 1 & شرايط كشت \\
\hline$\cdot|\cdots \cdot|$ & $\% \cdots \Delta$ & $\cdot / \cdot \Delta$ & /..r & .1 .4 & $\cdot / 4 F$ & . & $\cdot / M$ & II/ar &.$/ .0$ &.$/ 9 \mathrm{~V}$ & .1 .0 & זו/. & $\cdot \mid \Delta f$ &.$/ . .1$ & r & خطاى اول \\
\hline$\cdot|\cdots|^{* *}$ & $1 \ldots 9^{* * *}$ &.$/ . \cdot 1^{* * n}$ & $\cdot / \cdot+r^{* * * *}$ & $\cdot / \cdot Y^{\text {wask }}$ & $\cdot / \mu \cdot *$ & $\cdot|\ldots|^{* * *}$ &.$/ 19^{* *}$ & $1 F / \mathrm{Nq}^{* * * *}$ & $\cdot /\left.r\right|^{* *}$ & $\cdot / V \varphi^{* w}$ & $\cdot / r^{* * *}$ & $\cdot / \mu^{* *}$ & $1 / 4 \Lambda^{\text {*te }}$ & $\cdot / \cdot \gamma^{* *}$ & 90 & لاين \\
\hline$\cdot|\cdots|^{* * *}$ &.$/ \ldots r^{\omega *}$ & $\cdot /\left.\cdot \cdot\right|^{* * * a s}$ & $\cdot / \cdot r^{*}$ &.$/ 1^{w}$ & $\cdot \pi r^{\| *}$ & $\cdot|\cdots|^{\text {*t: }}$ & $\cdot / \cdot \gamma^{\omega *}$ & $\Delta / / V^{* N a k}$ & $\cdot / \cdot \varphi^{* *}$ & $\cdot / r \varphi^{* * *}$ & $\cdot / \cdot \omega^{* * *}$ & $\cdot|r|^{* * *}$ & 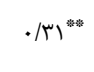 & $\cdot / \cdot 0^{* *}$ & 90 & لاين ×شترايط \\
\hline$\cdot|\cdots \cdot|$ & . &. $\mid \cdots 1$ &.$/ . .1$ &.$/ \cdots r$ &.$/ N F$ & $\cdot|\cdots \cdot|$ & $\cdot / \cdot 1$ &.$/ 199$ & . & r. &.$/ .1$ & .1 .9 & g & 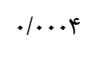 & & خطاى دوم \\
\hline$r F / . r$ & $r 8 / 11$ & $10 / q \pi$ & $\Delta S / F F$ & $s / \Lambda$. & $\Delta T / T A$ & $10 / r$. & $11 / 94$ & $10 / 4 \%$ & Q/vq & $1 r / r q$ & $\mathrm{~V} / \mathrm{WV}$ & $11 / \% 9$ & $N / T V$ & $r \Delta / q \gamma$ & & ضريب تغييي \\
\hline
\end{tabular}


جدول سـ هاى كنترل كننده براى صفات كياهجهاى در شرايط نرمال و كمبود نيتروثن در برنج Table 3. QTL controlling for seedling characteristics in normal conditions and a lack of nitrogen in rice

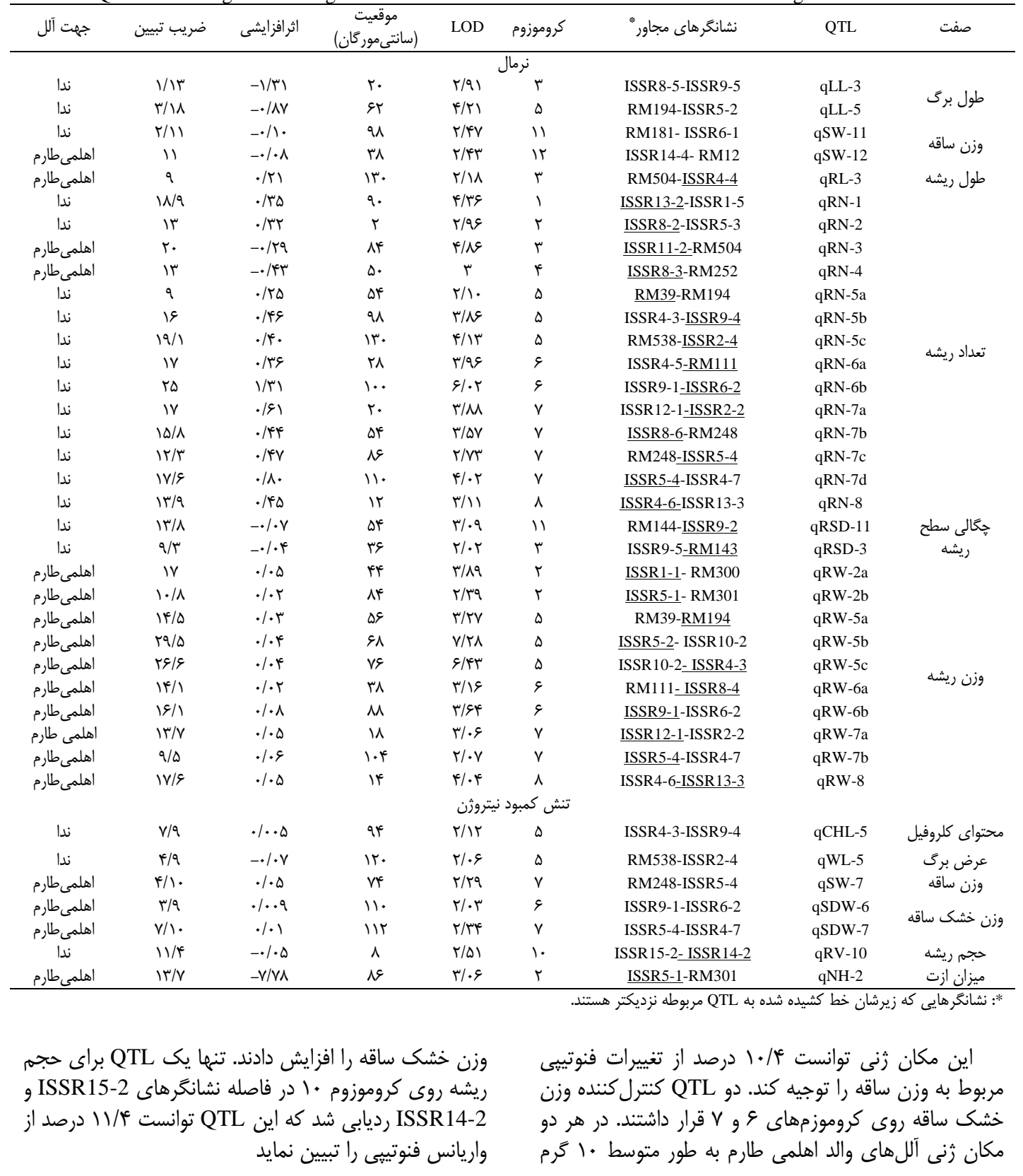



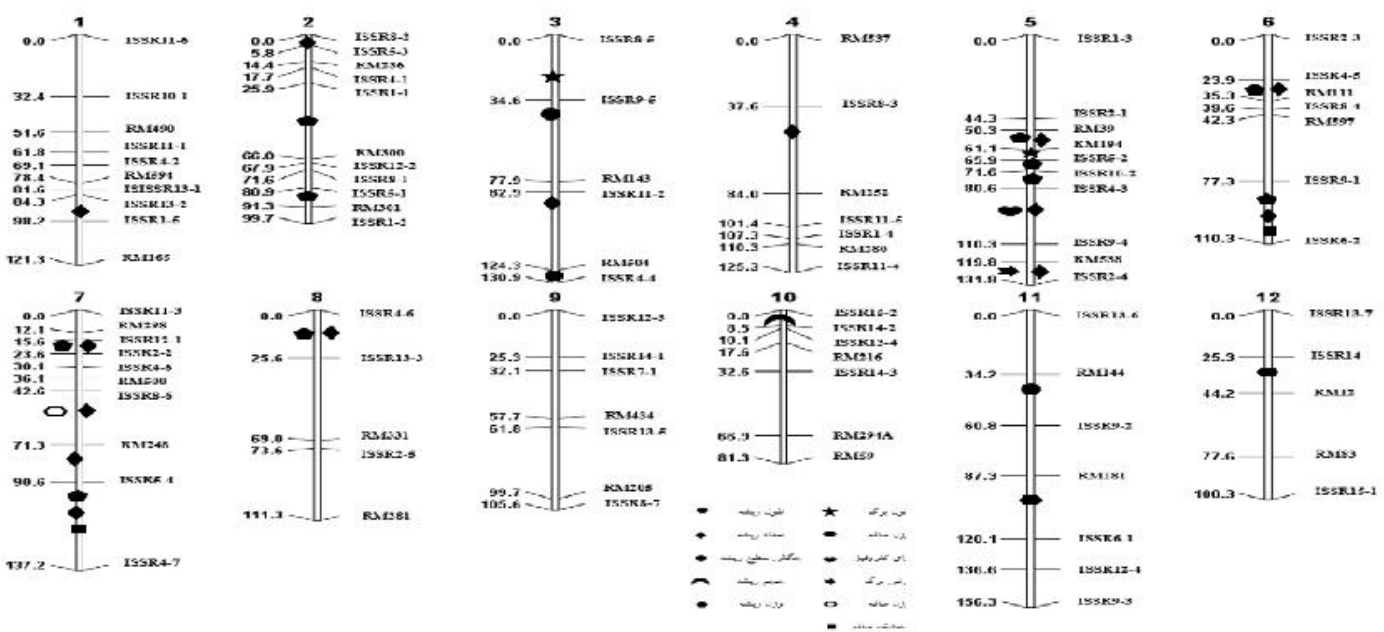

شكل ץ- - هاى كنترل كننده صفات مرتبط با كمبود نيتروثن در لاينهاى خالص نوتركيب حاصل از تلاقى ارقام اهلمى طارم و ندا در مرحله كَياهجهاى لاين

Figure 3. QTLs controlling nitrogen deficiency traits in pure recombinant lines resulting from the crossing of AhlmieTarom and Neda cultivars in seedling stage

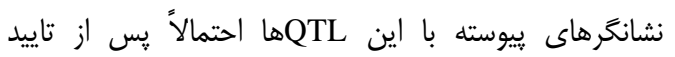

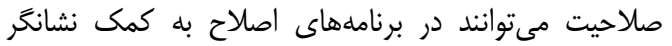
جهت ززينش لاينهاى برتر و انتقال آللهاى مطلوب استفاده

كردند.

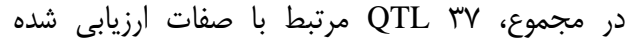
شناسايى شد. برخى از هاى شناسايى شده از جمله و qRW-5b qRN-6b qRN-5c aRN-3 ، qRN-1

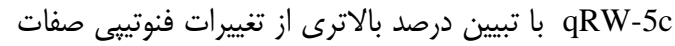

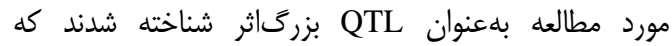


1. Ahmadi Shad, M.A. A A Ebadi, M.M Sohani, H Samizadh Lahiji and M. Hosseini Chaleshtori. The Assessment of Genetic Variation of Rice (Oryza Sativa L.) Recombinant Lines Based On Some of Quantitative and Qualitative Traits. Journal of Crop Breeding, 10(26): 166-172.

2. Fang, P. and P. Wu. 2001. QTL $\times$ N-level interaction for plant height in rice (Oriza Sativa L.). Plant and Soil, 236: 237-242.

3. Feng, Y., L.Y. Cao, W.M. Wu, X.H. Shen, X.D. Zhan, R.R. Zhai, R.C. Wang, D.B. Chen and S.H Cheng. 2010. Mapping QTLs for nitrogen-deficiency tolerance at seedling stage in rice (Oryza sativa L.). Plant Breeding, 129: 652-656.

4. Good, A.G., A.K. Shrawat and D.G. Muench. 2004. Can less yield more? Is reducing nutrient input into the environment compatible with maintaining crop production? Trends in Plant Science, 9, 597605.

5. Han -Hua, T.H., M. Han-Wei, Y. Xin-Qiao, X. Xiao-Yan and L. Ming-Shou. 2006. Identification of Related QTLs at Late Developmental Stage in Rice (Oryza sativa L.) Under Two Nitrogen Levels. Acta Genelica Sinica, 33 (5), 458-467.

6. Hu, S., D.L. Zeng, W. Su, X.Y. Shi, W.J. Ye, G.J. Dong, L. Zhu, J. Hu, Q. Qian and L.B. Guo. 2012. QTL analysis of nitrogen content of plant shoot under two nitrogen conditions in rice (Oryza sativa L.). Journal of Agronomy and Crop Science, 6(12): 1737-1744.

7. Ihizuka, Y. and A. Tanaka. 1953. Studies on the developmental processes view point of plant growth stage and root age. Bull. National Agricultural Science, 16: 19-150.

8. Katouzi, M., S. Navabpour, A. Yamchi, S. Ramezanpour, and H. Sabouri. 2017. Identification of Genes Controlling Seedling Stage Traits in Iranian RiceRecombinant Lines under Drought Stress Conditions. Journal of Crop Breeding, 9(21): 1-9.

9. Li, Z.K. 2001. QTL mapping in rice: afew critiical consideration. In Khush G.S., D.S. Brar, and B. Hardy (Eds.). Genetics IV. Science publisher Inc, 153-171.

10. Lian, X., Y. Xing, H. Yan, G. Xu, X. Li and Q. Zhang. 2005. QTLs for low nitrogen tolerance at seedling stage identified using a recombinant inbred line population derived from an elite rice hybrid. Theoretical and Applied Genetics, 112: 85-96.

11. Peng, M., C. Hannam, H. Gu, Y.M. Bi and S.J. Rothstein. 2007. A mutation in NLA, which encodes a RING-type ubiquitin ligase, disrupts the adaptability of Arabidopsis to nitrogen limitation. Plant Journal, 50: 320-337.

12. Richard-Molard, C., A. Krapp, F. Brun, B. Ney, F. Daniel-Vedele, and S. Chaillou. 2008. Plant response to nitrate starvation is determined by $\mathrm{N}$ storage capacity matched by nitrate uptake capacity in two Arabidopsis genotypes. Journal of Experimental Botany, 59: 79-91.

13. Saghi Maroof, M.A., R.M. Biyashev, G.P. Yang, Q. Zhang and R.W. Allard. 1994. Extraordinarily polymorphic microsatellites DNA in barely species diversity, choromosomal location, and population dynamics. Proceedings of the National Academy of Sciences, 91: 5466-5570.

14. Tahmoorespoor, M. 2010. Assessment relationship between GH and STAT5A genes polymorphism and estimated breeding value (EBV) of growth traits in Baluchi sheep. Ferdowsi University of Mashhad, Iran, $54 \mathrm{pp}$.

15. Wei, D., K. Cui, G. Ye, J. Pan, J. Xiang, J. Huang and L. Nie. 2012. QTL mapping for nitrogen-use efficiency and nitrogen-deficiency tolerance traits in rice. Plant Soil, 359: 281-295.

16. Yu, W., S. Yong-Jian, C. Deng-Yin and Y. Si-Bin. 2009. Analysis of Quantitative Trait Loci in Response to Nitrogen and Phosphorus Deficiency in Rice Using Chromosomal Segment Substitution Lines. Acta Agronomic Sinica, 35(4): 580-587.

17. Yoshida, S., D.A. Forno, J.H. Cock and K.A. Gomez. 1976. Laboratory manual for physiological studies of rice. IRRI, Los banos, philipines, $83 \mathrm{pp}$

18. Manly, K.F. and J.M. Olson. 1999. Overview of QTL mapping software and introduction to Map Manager QTL. Mammalian Genom, 10: 327-334. 


\title{
Identification of QTLs Related to Root and Shoot Traits in Rice under Nitrogen Deficiency Conditions
}

\author{
Sharifeh Mohammadalegh ${ }^{1}$, Hossein Sabouri ${ }^{2}$, Ali Sattarian ${ }^{3}$, Abbas Biabani $^{3}$ and $^{4}$ \\ Abdollatif Gholizadeh ${ }^{4}$ \\ 1- M.Sc. Student of Biotechnology of Agriculture Science and Natural Resource, Gonbad Kavous University \\ 2- Associate Professor Department of Plant Production, College of Agriculture Science and Natural Resource, \\ Gonbad Kavous University (Corresponding author: hos.sabouri@gmail.com) \\ 3- Associate Professor Department of Biology, College of Agriculture Science and Natural Resource, Gonbad \\ Kavous University \\ 4- Assistant Professor Department of Plant Production, College of Agriculture Science and Natural Resource, \\ Gonbad Kavous University \\ Received: July 30, $2015 \quad$ Accepted: September 10, 2017

\begin{abstract}
In order to detect the QTL associated with nitrogen-deficiency tolerance at seedling stage, a population of 96 lines derived from Ahlami Tarom and Neda cross were studied at 2014. For genetic linkage map construction, 30 SSR and 15 ISSR makers were used. The resulted linkage map covered $1411.3 \mathrm{cM}$ of rice genome with an average of $15.34 \mathrm{cM}$ distance between two markers. A total of 37 QTLs were identified for the traits under study. qRV-10 under conditions of nitrogen deficiency explained $11.4 \%$ of the phenotypic variations of root volume. QTLs controlling root number ( $\mathrm{qRN}-3$ and $\mathrm{qRN}-6 \mathrm{~b})$ and root weight (qRW-5b and $\mathrm{qRW}-5 \mathrm{c}$ ) under normal conditions were detected in the major stress that this is justified QTL accounted for over 20 percent of phenotypic changes. Markers linked to the QTL as markers linked to traits are likely to be used for marker-assisted selection in breeding programs.
\end{abstract}

Keywords: Nitrogen deficiency, Quantitative Traits Loci, Rice, Seedling stage 